\section{MAPEAMENTO EPIDEMIOLÓGICO DAS \\ PARASITOSES INTESTINAIS EM SEIS \\ BAIRROS DO MUNICÍPIO DE ARARAQUARA/SP}

Viviane Ferre de Souza*

Gisele de Lucca*

Fernanda de Freitas Anibal**

Introdução

As parasitoses intestinais constituem um grave problema de saúde pública. Fato este oriundo do precário saneamento básico e das baixas condições sócio-econômicas. Os indivíduos pertencentes às classes menos favorecidas economicamente são acometidos com a alta prevalência por parasitoses intestinais. O quadro de desnutrição comum a estes indivíduos acaba agravando o parasitismo e dificulta uma melhor resposta de seus organismos contra os patógenos (COSTA-MACEDO, 1998).

No Brasil, grandes inquéritos coproparasitológicos foram realizados até a década de 70 . Nos últimos anos, contamos apenas com trabalhos isolados, que pela diversidade geográfica, social, econômica e cultural do País, nem sempre podem ser comparados. Entretanto, nas regiões com infra-estrutura urbana deficiente, os inquéritos mostram que pelo menos metade das crianças residentes encontra-se parasitadas (GROSS et al., 1989; SANTANA, ALENCAR, ROUQUAYROL, 1994)

O estudo epidemiológico de quaisquer parasitas intestinais proporciona diversas informações, especialmente quanto à insalubridade do meio, nível e extensão do saneamento básico de uma região e também quanto aos hábitos higiênicos da população em estudo. Em decorrência dos efeitos deletérios à saúde dos indivíduos e, sobretudo, das repercussões econômicas, vários programas têm sido dirigidos para o controle das parasitoses intestinais em

* Biomédica formada pelo Centro Universitário de Araraquara - Uniara.

** PhD, Professora de Parasitologia Clínica do Centro Universitário de Araraquara - Uniara e Professora de Imunologia e Microbiologia das Faculdades Integradas Fafibe e Faculdades São Luís de Jaboticabal. diferentes países, mas, infelizmente, constata-se um descompasso entre o êxito alcançado nos países mais desenvolvidos e aquele verificado nas economias mais pobres. Além, do custo financeiro das medidas técnicas, a falta de projetos educativos com a participação da comunidade dificultam a implementação das ações de controle (FANUCHI et al., 1984; PEDRAZZANI et al., 1989; VINHA, MARTINS, 1981). Nos países subdesenvolvidos, as parasitoses intestinais atingem índices de até $90 \%$, ocorrendo um aumento significativo da freqüência à medida que piora o nível sócio econômico (REY, 2001).

A principal fonte de parasitas que contribui para a contaminação do ser humano se encontra no solo e na água, sendo que o mesmo contribui para contaminar os alimentos e o meio ambiente (REY, 2001). O lançamento dos dejetos humanos “in natura”, através de redes de esgotos e estação de tratamento nos rios e riachos, promove a contaminação do meio por ovos, cistos e larvas que irão promover a infecção de novos hospedeiros

Dentre as principais patogenias intestinais causadas por parasitas podemos destacar a Giardíase que é uma infecção do intestino delgado causada pela Giardia lamblia, um protozoário flagelado. A infecção ocorre através de cistos eliminados nas fezes, podendo ocorrer diretamente, entre as crianças ou parceiros sexuais, ou indiretamente, através de alimentos ou de água contaminados (REY, 2001);

Amebíase: é uma infecção do intestino grosso causada pela Entamoeba histolytica. A transmissão ocorre por meio do contato com fezes infectadas através de cistos (REY, 2001). Além da E. histolytica, outras amebas são freqüentemente encontradas nas fezes de crianças e adultos. Embora não seja patogênica, a presença destes protozoários no organismo humano representa o contato direto ou indireto com material contaminado por coliformes fecais, o que é muito preocupante quando pensamos na possibilidade de aumentarmos a chance de adquirir parasitas mais agressivos, são exemplos: Entamoeba coli, Iodamoeba butschilli, Endolimax nana;

Teníase: é uma alteração provocada pela presença da forma adulta da Taenia solium ou da Taenia saginata (vulgarmente denominada por solitária) no intestino delgado humano (NEVES,1997). A contaminação ocorre através da ingestão de carne mal cozida contaminada com cisticerco de ambas as tênias (REY, 2001);

Himenolepíase: é uma infecção do intestino delgado, principalmente íleo e jejuno, causada pelo Hymenolepis nana ("tênia anã"), sendo o menor e mais comum dos cestódeos que ocorrem no homem (NEVES,1997). O mecanismo mais freqüente de transmissão é a ingestão de ovos presentes nas mãos ou em alimentos contaminados (NEVES, 1997);

Ascaridíase: é uma infecção do intestino delgado do homem, produzida pelo nematódeo Ascaris lumbricoides, parasita de distribuição cosmopolita, 
vulgarmente denominado "lombriga", sendo o maior nematódeo intestinal do homem (CIMERMAN, CIMERMAN, 1999). É adquirida pela ingestão de ovos infectantes em alimentos contaminados (CIMERMAN, CIMERMAN, 1999).

Ancilostomíase: é a infecção de seres humanos por nematódeos da família Ancylostomatidae, que compreende duas espécies de importância, o Ancylostoma duodenale e o Necator americanus (CIMERMAN, CIMERMAN, 1999). Regiões úmidas, quentes e contato direto da pele com solo contendo larvas infectantes em áreas sem saneamento básico (LEVENTHAL, CHEADLE, 2000) são modos de aquisição;

Estrongiloidíase: infecção das criptas da mucosa duodenal, principalmente nas glândulas de Lieberkühn e porção superior do jejuno, causada em geral pelo Strongyloides stercoralis (NEVES, 1997). A heteroinfecção é a via de infecção mais freqüente e ocorre através da penetração de larvas infectantes na pele dos pés, mãos, espaços interdigitais e nádegas (CIMERMAN, CIMERMAN, 1999);

Enterobíase: infecção intestinal associada ao ceco, apêndice e ânus, popularmente chamada de "oxiúro", causada pelo parasita Enterobius vermicularis. É uma das parasitoses com maior poder de infecção, onde a contaminação ocorre por inalação de ovos presentes na poeira de dormitórios, colégios, habitações coletivas e roupas de cama, podendo ainda ocorrer uma auto-infecção ânus-boca, principalmente em crianças e portadores de necessidades especiais (CIMERMAN, CIMERMAN, 1999);

Trichiuríase: infecção da mucosa do ceco pelo Trichuris trichiura, podendo ser visto também no apêndice, cólon e íleo (NEVES, 1997).

A identificação dos principais parasitas intestinais responsáveis pelas doenças parasitárias em moradores de seis bairros do município de Araraquara/ SP, permite a participação de profissionais da saúde na melhoria da qualidade de vida destas pessoas neste município. Deste modo, identificamos os parasitas intestinais em exames de fezes de moradores de seis bairros do município de Araraquara/SP, além de determinar o perfil de focos endêmicos, pesquisando na população a incidência e prevalência das principais doenças parasitárias intestinais que acometem pessoas em diversas idades.

\section{Casuística e métodos}

\section{Indivíduos incluídos neste estudo}

Foram analisados 203 laudos de exames de fezes positivos para alguma espécie parasitária, de moradores de seis bairros do município de Araraquara/ $\mathrm{SP}$, de ambos os sexos e diversas idades, no período de março a agosto de 2004.

\section{Diagnóstico Laboratorial}

Os exames foram realizados no laboratório de Parasitologia Clínica, no laboratório da Santa Casa de Misericórdia de Araraquara/SP.

\section{Técnicas utilizadas}

\section{Método direto a fresco}

Princípio - exame direto de fezes, utilizado na pesquisa de cistos, trofozoítos de protozoários e ovos de helmintos.

Técnica - a pesquisa deve ser feita com objetiva de médio e grande aumento, entre lâmina e lamínula, diluindo, se necessário, em solução salina (CIMERMAN, CIMERMAN, 1999).

Método de Faust e colaboradores

Princípio - centrífugo-flutuação em sulfato de zinco a 33\% com densidade 1.180 (CIMERMAN, CIMERMAN, 1999).

Técnica - diluir $10 \mathrm{~g}$ de fezes em $20 \mathrm{ml}$ de água filtrada; filtrar através de gaze dobrada em quatro, num tubo de ensaio; centrifugar por 1 minuto a 1500 rpm; desprezar o sobrenadante, ressuspender a matéria depositada em água e centrifugar até que o sobrenadante fique claro; desprezar o sobrenadante e adicionar $2 \mathrm{ml}$ da solução de sulfato de zinco; repetir a centrifugação. As formas evolutivas dos parasitas presentes estarão numa película flutuante na superfície do líquido; a mesma é recolhida com uma alça de platina, colocada em uma lâmina coberta por uma lamínula, para a visualização ao microscópio (NEVES, 1997).

\section{Método de Hoffmann Pons \& Janer ou Lutz}

Princípio - sedimentação espontânea em água, utilizada para evidenciar ovos pesados de helmintos, ovos leves de helmintos e cistos de protozoários, quando a sedimentação for por um período de 24 horas (CIMERMAN, CIMERMAN, 1999).

Técnica - adicionar cerca de $2 \mathrm{~g}$ de fezes em um béquer ou borrel, com cerca de $5 \mathrm{ml}$ de água, e triturar bem com bastão de vidro. Acrescentar mais 20 $\mathrm{ml}$ de água, filtrar a suspensão para um cálice cônico de $200 \mathrm{ml}$ de capacidade, por intermédio de gaze cirúrgica dobrada em quatro. Essa suspensão de fezes é deixada em repouso de 2 a 24 horas. Colocar o sedimento entre lâmina e lamínula para a visualização ao microscópio (NEVES, 1997).

\section{Resultados e discussão}

Caracterização da incidência de parasitas intestinais em moradores de seis bairros do município de Araraquara/SP

As análises dos prontuários dos achados laboratoriais demonstraram a incidência de doze espécies de parasitas intestinais nos seis bairros analisados. 
Destes doze parasitas, nove são patogênicos. Dentre os patogênicos, a Giardia lamblia foi o mais encontrado (Fig. 1). Estes dados corroboram com alguns achados da literatura, onde os autores relataram que 20 a $25 \%$ da população do Estado de São Paulo apresentavam infeç̧ão pela Giardia lamblia. (FANUCHI et al, 1984).

Em relação aos helmintos, observamos que a Hymenolepis nana foi o mais encontrado nas amostras analisadas no Laboratório de Análises Clínicas da Santa Casa de Misericórdia de Araraquara/SP.

Esses dados diferem de alguns já demonstrados na literatura, onde o Ascaris lumbricoides é o helminto de maior incidência na população (COSTAMACEDO, COSTA, ALMEIDA, 1999). Essa alta incidência parasitária pode estar relacionada com as condições deficientes tanto de moradia quanto monetária dos moradores desses bairros, visto que a grande maioria dos bairros analisados é da periferia do município.

\section{Incidência de Parasitoses nos Bairros de Araraquara}

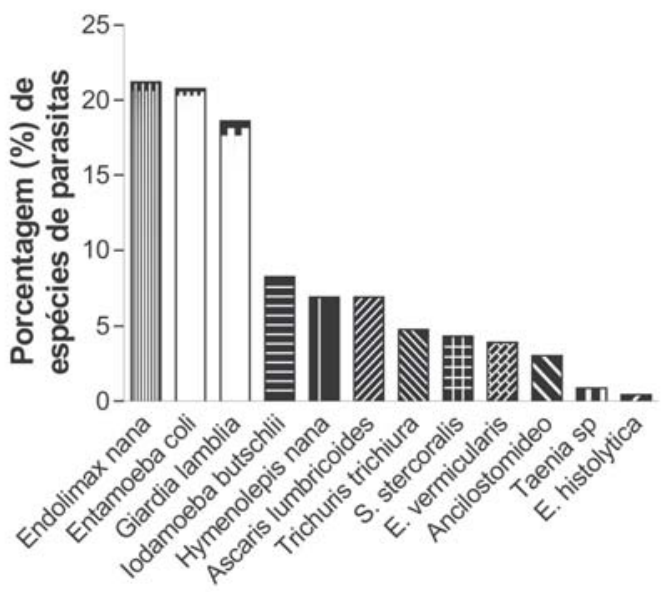

Figura 1 - Representação gráfica do número relativo (\%) da incidência das parasitoses intestinais em moradores de seis bairros do município de Araraquara-SP, onde foram encontradas doze espécies diferentes de parasitas intestinais, dentre os quais nove são patogênicos.
Distribuição da incidência de diversas parasitoses intestinais em indivíduos sem distinção de sexo

Os dados analisados dos achados laboratoriais demonstram a similaridade do acometimento das parasitoses intestinais em ambos os sexos, onde o sexo feminino apresentou 107 (52,71\%) casos positivos e o sexo masculino 96 (47,29\%) casos, dentre os 203 laudos analisados. Estes dados precisam ser melhor investigados, devido ao fato de não termos limitado os números de amostras estudadas em cada sexo.

Distribuição da incidência de diversas parasitoses intestinais nos seis bairros analisados do município de Araraquara/SP

A análise realizada nos seis bairros do Município de Araraquara/SPrevelaram que o bairro Selmi Dei obteve o maior índice de acometimento das diversas parasitoses intestinais, totalizando 96 (45,81\%) casos positivos (Fig. 2). Estes dados indicam que uma fonte para as parasitoses poderia ser os córregos que cortam o bairro, que devem ser ou estar contaminados por dejetos humanos e que são utilizados para a obtenção de água para uso doméstico, além de irrigar hortas que produzem produtos de consumo para os moradores do bairro.

O bairro Parque São Paulo vem logo após, com um total de 58 (28,57\%) casos, seguido dos bairros Jardim Iguatemi, com 21 (10,35\%) casos; Cecap, com 14 (6,90\%) casos; Vila Xavier, com 13 (6,40\%) casos, e, por fim, o bairro que revelou menor índice de acometimento das parasitoses intestinais, o Jardim América, com 4 $(1,97 \%)$ casos positivos.

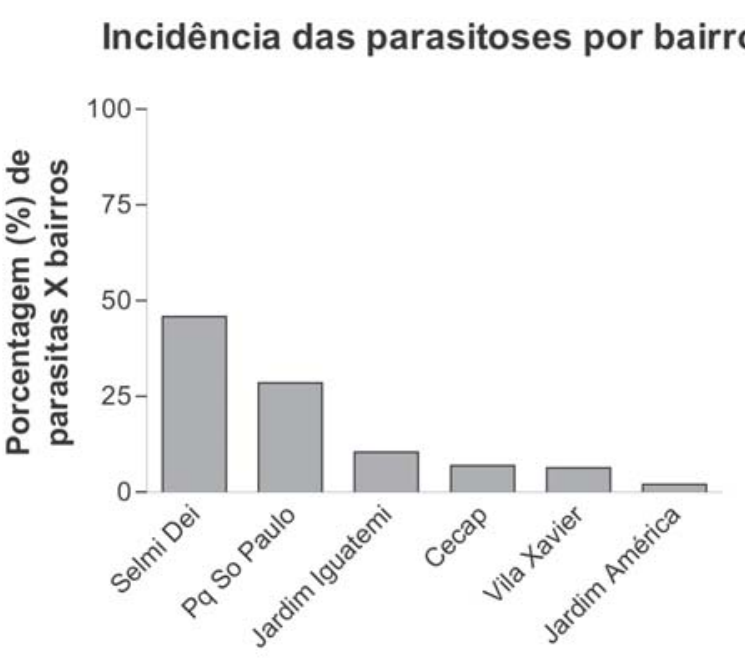

Figura 2 - Distribuição das parasitoses intestinais em número relativo (\%) nos seis bairros do município de Araraquara-SP, caracterizando o bairro Selmi Dei com a maior incidência das parasitoses intestinais, e o bairro Jardim América com a menor incidência. 
Caracterização da incidência de diversas parasitoses intestinais em moradores do bairro Selmi Dei

Os dados laboratoriais analisados constataram a incidência de dez espécies de parasitas intestinais no bairro Selmi Dei, dentre os quais sete são patogênicos. Levando em consideração os parasitas intestinais patogênicos, que constituem importância clínica, a Giardia lamblia encontra-se prevalente com 23 (21,70\%) casos. Podemos encontrar logo após Ascaris lumbricoides, com 9 (8,49\%) casos; em seguida encontra-se Hymenolepis nana, com 8 (7,55\%) casos; Ancilostomídeo, com 6 (5,66\%) casos; Strongyloides stercoralis, com 3 (2,83\%) casos; Trichuris trichiura, com 2 (1,89\%), e, por último, Taenia sp, com $1(0,94 \%)$ caso positivo.

Caracterização da incidência de diversas parasitoses intestinais em moradores do bairro Parque São Paulo

No bairro Parque São Paulo, a análise dos achados laboratoriais demonstraram a incidência de onze espécies de parasitas intestinais, sendo que, destes, oito são patogênicos. Dentre as patogênicas, destacamos a Giardia lamblia como parasita de maior incidência, com 10 (15,15\%) casos, seguida de Ascaris lumbricoides, com 6 (9,09\%) casos; Hymenolepis nana e Enterobius vermicularis, com 5 (7,58\%) casos; Strongyloides stercoralis e Trichuris trichiura, com 4 (6,06\%), e, por fim, Ancilostomídeo e Taenia sp, com 1 (1,51\%) caso positivo.

Caracterização da incidência de diversas parasitoses intestinais em moradores do bairro Jardim Iguatemi

No bairro Jardim Iguatemi, a análise dos achados laboratoriais demonstraram a incidência de oito espécies de parasitas intestinais, sendo que, destes, seis são patogênicos. Dentre os patogênicos, a Giardia lamblia encontrase à frente, com 5 (21,73\%) casos, seguida de Trichuris trichiura, com 3 (13,04\%) casos; Hymenolepis nana e Enterobius vermicularis, com 2 (8,70\%) casos, e caracterizando a menor incidência, observamos Strongyloides stercoralis e Ascaris lumbricoides, com 1 (4,35\%) caso positivo.

Caracterização da incidência de diversas parasitoses intestinais em moradores do bairro Cecap

No bairro Cecap, a análise dos achados laboratoriais demonstrou a incidência de cinco espécies de parasitas intestinais, destes, três são patogênicos. Dentre os patogênicos, ocorre a prevalência de Strongyloides stercoralis, com $2(14,29 \%)$ casos, seguido de Giardia lamblia e Trichuris trichiura, com 1 (7,14\%) caso positivo.

Caracterização da incidência de diversas parasitoses intestinais em moradores do bairro Vila Xavier

No bairro Vila Xavier, a análise dos achados laboratoriais demonstrou a incidência de sete espécies de parasitas intestinais, sendo quatro são patogênicos.
Dentre os patogênicos, a Giardia lamblia obteve maior incidência, com 2 (11,76\%) casos, seguida de Trichuris trichiura, Hymenolepis nana e Entamoeba histolytica, com 1 (5,88\%) caso positivo.

Caracterização da incidência de diversas parasitoses intestinais em moradores do bairro Jardim América

No bairro Jardim América, a análise dos achados laboratoriais demonstrou a incidência de três espécies de parasitas intestinais, destes, apenas um não era patogênico. Dentre os patogênicos, Giardia lamblia e Enterobius vermicularis apresentaram a mesma incidência, com 2 (40,0\%) casos positivos.

\section{Conclusão}

Após a análise dos prontuários dos laudos laboratoriais de seis bairros assistidos pela Santa Casa de Misericórdia de Araraquara/SP, concluímos que: - dentre os parasitas intestinais patogênicos, encontrados nos seis bairros analisados, a Giardia lamblia foi o mais freqüente.

- Dentre os parasitas intestinais não patogênicos, encontrados nos seis bairros analisados, a Endolimax nana foi o mais encontrado, revelando a exposição desses pacientes a contaminantes fecais.

- Dentre os sexos analisados, não houve diferenças significativas no acometimento pelas parasitoses intestinais.

- Dentre os seis bairros analisados, o Selmi Dei foi o bairro de maior incidência de parasitoses intestinais, com 45,81\% do total analisado; enquanto o Jardim América foi bairro de menor incidência, com 1,97\% do total analisado.

- A alta incidência parasitária pode estar relacionada com as condições deficientes tanto de moradia quanto monetária dos moradores desses bairros, visto que a grande maioria dos bairros analisados é da periferia do município, o que propicia a exposição e contaminação pelos diversos parasitas encontrados.

Assim, concluímos que o mapeamento epidemiológico das parasitoses intestinais no município de Araraquara/SP fornece parâmetros geográficos que facilitam a atuação dos agentes de saúde nos focos mais endêmicos, proporcionando a diminuição da incidência dessas doenças na cidade de Araraquara/SP, assim como a adequação de medidas profiláticas.

\section{Referências:}

CIMERMAN, B.; CIMERMAN, S. Parasitologia humana e seus fundamentos gerais. São Paulo: Editora Atheneu, 1999.

COSTA-MACEDO, L.M. et al. Enteroparasitoses em pré-escolares de comunidades favelizadas da cidade do Rio de Janeiro, Brasil. Caderno de Saúde Pública. Rio de Janeiro, v.14, p.851-855, 1998. 
COSTA-MACEDO, L.M.; COSTA, M.C.E.; ALMEIDA, L.M. Parasitismo pelo Ascaris lumbricoides em crianças menores de dois anos em comunidades abertas do Rio de Janeiro. Caderno de Saúde Pública. Rio de Janeiro, v.15, p.173-178, 1999.

FANUCHI, J.N. et al. Contaminação da água altos índices de Giardíase. Jornal de Pediatria, Rio de Janeiro, v.56, p.117-119, 1984.

GENDREL, D.et al. Precocite d'apparition du parasitisme intestinal Chez le nourisson em Afrique Equatoriale. In: Annales de Pédriatrie, Coimbra, Portugal v.30, p.453-456, 1983.

GROSS, R. et al. The impact of improvement of water supply and sanition facilities on diarrhea intestinal parasites: a brazilian experience whith children in two low-income urban communities. Revista de Saúde Pública. São Paulo, v.23, p.214-220, 1989.

LEVENTHAL, R.; CHEADLE, R. Parasitologia médica - Texto e atlas. 4.ed. São Paulo: Editorial Premier, 2000.

NEVES, David Pereira et al. Parasitologia Humana. 9. ed. São Paulo: Atheneu, 1997.

NEVES, David Pereira et al. Parasitologia Humana. 10. ed. São Paulo: Atheneu, 2002.

PEDRAZZANI, E.S.et al. Helmintoses intestinais. III- Programa de Educação e Saúde em Verminose. Revista de Saúde Publica. São Paulo, v.23, p189-195, 1989.

REY, Luís. Parasitologia. 3.ed. Rio de Janeiro: Guanabara Koogan S.A., 2001 .

SANTANA, L. R.; ALENCAR, M. J. M.; ROUQUAYROL, M. Z.

Poliparasitismo Intestinal e residiva de enteroparasitoses em crianças de tenra idade. Revista Brasileira de Análises Clínicas. São Paulo, v.26, p.5052, 1994.

VINHA, C.; MARTINS, M.R.S. Parasitoses intestinais entre escolares.

Jornal de Pediatria, Rio de Janeiro, v.50, p.79-84, 1981.

\section{Resumo:}

No Brasil, grandes inquéritos coproparasitológicos foram realizados até a década de 70. Nos últimos anos, contamos apenas com trabalhos isolados, que nem sempre podem ser comparados. Entretanto, nas regiões com infra-estrutura urbana deficiente, os inquéritos mostram que grande parte da população residente encontra-se parasitada (Gross et al., 1994: Santana et al., 1994).

O estudo epidemiológico de quaisquer parasitas intestinais proporciona diversas informações, especialmente quanto à insalubridade do meio, nível e extensão do saneamento básico de uma região e também quanto aos hábitos higiênicos da população em estudo. Desta forma, neste estudo tivemos como objetivo caracterizar a incidência de parasitoses intestinais em moradores de seis bairros do município de Araraquara/SP.

Nossos resultados demonstraram a grande incidência de parasitas intestinais. A Endolimax nana foi o parasita intestinal mais encontrado. Embora não sendo patogênico, sua existência revela a exposição destas pessoas a contaminantes fecais. A Giardia lamblia foi o parasita intestinal patogênico mais encontrado. Houve similaridade de acometimento das parasitoses tanto no sexo feminino quanto no masculino. O bairro Selmi Dei obteve o maior índice dessas parasitoses, caracterizando-se como o bairro de maior preocupação para saúde pública do município de Araraquara/SP.

\section{Palavras-chave:}

Parasitoses Intestinais, Bairros do Município de Araraquara/SP, Parasitas Patogênicos. 